\title{
Adaptação de lentes de contato pós-trauma ocular
}

\author{
Contact lens fitting after ocular trauma
}

$\begin{array}{llll}\text { Heryberto } & \text { da } & \text { Silva } & \text { Alvim } \\ \text { Leonardo } & \text { Torqueti } & \text { Costa }^{1} \\ \text { Rogério } & \text { de Almeida } & \text { Tárcia }^{2} \\ \text { Lourival } & \text { Franco de } & \text { Sá } & \text { Filfico }^{3}\end{array}$

Residentes de Oftalmologia do Hospital São Geraldo, Hospital das Clínicas da Universidade Federal de Minas Gerais - UFMG.

${ }^{2}$ Oftalmologista, Fellow do Serviço de Ecografia do Hospital São Geraldo, Hospital das Clínicas da Universidade Federal de Minas Gerais - UFMG.

${ }^{3}$ Professor Adjunto, Faculdade de Medicina, Universidade Federal de Minas Gerais. Chefe do Serviço de Lentes de Contato, Hospital São Geraldo.

Endereco para correspondência: Dr. Lourival Franco de Sá, Hospital São Geraldo - Serviços de Lentes de Contato - Av. Alfredo Balena, $190-3^{\circ}$ andar Belo Horizonte (MG) - CEP 31130-100

Recebido para publicação em 19.04.2002 Aceito para publicação em 16.12.2002

Nota Editorial: Pela análise deste trabalho e por sua anuência na divulgação desta nota, agradecemos ao Dr. Ricardo Lewinsky.

\begin{tabular}{|l|}
\hline R E s u M o \\
\hline Objetivos: Descrever os achados referentes à adaptação de lentes de \\
contato nos casos de trauma ocular nos últimos 6 anos no Hospital São \\
Geraldo, identificando os mecanismos dos traumas, os parâmetros das \\
lentes de contato prescritas, e por fim avaliar quantitativamente a \\
melhora da acuidade visual com o uso destas. Métodos: Revisão dos \\
prontuários de pacientes atendidos no Serviço de Lentes de Contato \\
do Hospital São Geraldo nos últimos 6 anos. Os dados referentes à \\
idade, sexo, diagnóstico, doenças associadas, olho acometido, acuidade \\
visual pós-trauma, raio, curvatura e índice de permeabilidade ao oxigê- \\
nio das lentes são descritos. Resultados: O principal diagnóstico foi \\
perfuração, com 25 casos (44,6\%), o olho esquerdo estando acometido \\
em 25 casos (58,2\%). A AV pós-trauma corrigida mais freqüente foi $20 /$ \\
200 (20,9\%). A AV corrigida média após o uso das lentes de contato foi \\
de 20/20(14\%). O maior ganho em número de linhas na tabela de Snellen \\
ocorreu em seis pacientes (14\%) (5 linhas). O diâmetro médio das lentes \\
foi de 9,5 mm, o raio médio foi de 8,0 mm. A curvatura das lentes variou \\
de 37,00 D a 52,75 D. A maioria das lentes tinha DK 71. A adaptação foi \\
adequada em todos os casos. O intervalo médio entre o trauma e o início \\
da adaptação foi de 3,5 anos. Conclusão: As lentes de contato são parte \\
importante no tratamento tardio do trauma ocular, permitindo melhora \\
significativa da AV na maioria dos casos.
\end{tabular}

Descritores: Lentes de contato; Traumatismos oculares; Acuidade visual; Adaptação ocular

\section{INTRODUÇÃO}

$\mathrm{O}$ atendimento ao paciente vítima de trauma ocular não deve resumirse ao procedimento cirúrgico de correção das lesões (laceração corneana, facectomia, vitrectomia anterior, etc), ou ao tratamento clínico destas em sua fase aguda (irite, glaucoma, ceratite, etc). O trauma que resulta em perfuração do globo ocular comumente deixa seqüelas como leucoma corneano, afacia, astigmatismo regular e irregular. Estas condições por sua vez são causas de baixa acuidade visual (BAV) que muitas vezes são corrigidas com o uso de lentes de contato (LC). O presente estudo faz um perfil do paciente que foi atendido no Serviço de Lentes de Contato do Hospital São Geraldo entre os anos de 1995 a 2000, apontando aspectos epidemiológicos, o mecanismo do trauma e os parâmetros mais comuns de adaptação das lentes de contato no trauma. Avaliaremos a capacidade de reabilitação visual das lentes medida pela melhora da acuidade visual (AV), o que nos permitirá concluir se a prescrição destas dispensaria um novo procedimento cirúrgico se o objetivo deste for apenas recuperar visão. 


\section{MÉTODOS}

Estudou-se retrospectivamente os prontuários do Serviço de Lentes de Contato referentes aos últimos 6 anos (1995 a 2000). Apenas aqueles com história de trauma ocular não cirúrgico foram estudados, perfazendo um total de 43 pacientes. As variáveis estudadas foram: diagnóstico que motivou o encaminhamento, idade, sexo, doenças oculares concomitantes, olho acometido, as acuidades visuais pós-trauma e após o uso das lentes de contato. Foram avaliadas as especificações das lentes tais como valor dióptrico (D), diâmetro, raio de curvatura, curvatura em dioptrias e o índice de permeabilidade ao oxigênio (DK). O intervalo entre o trauma e a chegada do paciente ao Serviço de Lente de Contato, as complicações do seu uso, se houve troca das lentes por outras e os motivos da não indicação desta foram anotados neste estudo. A adaptação foi classificada como:

1) Adequada: se não havia sintomas, ou se estes quando presentes eram suportáveis.

2) Não adequada: presença de sintomas que impossibilitaram o uso regular das lentes.

A AV pós-trauma é aquela encontrada no momento da chegada do paciente no Serviço de Lentes de Contato, com sua melhor correção obtida na refração subjetiva. A tabela de Snellen foi utilizada para avaliar a AV. Quando esta era pior que 20/400 era então classificada em conta dedos ou visão de vultos junto ao rosto. A melhora da visão foi quantificada em número de linhas lidas a mais (Tabela de Snellen) em relação à $\mathrm{AV}$ antes da adaptação das lentes de contato.

\section{RESULTADOS}

A idade variou de 7 a 66 anos entre os homens, com uma média de 27 anos, e de 6 a 45 anos entre as mulheres, média de 22 anos. A média de idade de ambos os sexos foi de 25,7 anos com desvio-padrão de 14,1 . Os homens representaram 74,4\% dos casos. O diagnóstico mais comum foi afacia secundária a trauma - 16 casos $(37,21 \%)$ seguido por leucoma corneano com 14 casos $(32,56 \%)$ e por último astigmatismo corneano com 13 casos $(30,23 \%)$. Todos casos de afacia e de astigmatismo irregular foram causados por perfuração ocular assim como 9 dos 14 casos de leucoma. Portanto a perfuração foi o principal mecanismo de trauma que levou os pacientes ao Serviço de Lentes de Contato. No entanto este diagnóstico não foi utilizado pois não contribuiu para compreendermos o motivo da indicação da lente de contato. O olho esquerdo foi acometido em 25 casos $(58,2 \%)$, não houve casos de bilateralidade. Algumas alterações eram concomitantes no momento da adaptação das lentes: entrópio cicatricial secundário à queimadura química, luxação posterior do cristalino devido a trauma contuso, ceratocone e atrofia do epitélio pigmentar macular, todas estas ocorrendo apenas uma vez na amostra e em diferentes pacientes.

A AV pós-trauma variou de 20/15 (1 caso - 2,32\%) a visão de vultos junto ao rosto ( 1 caso - 2,32\%). A visão corrigida de $20 / 200$ foi a mais encontrada, com 9 casos $(20,93 \%)$ seguida por $8(18,61 \%)$ casos com AV de 20/60. Após a adaptação das lentes de contato a melhor AV obtida foi de 20/20 em 7 casos $(16,27 \%)$, porém a $\mathrm{AV}$ de $20 / 25$ foi a mais freqüente em uso de lente de contato, sendo observada em 8 pacientes $(18,61 \%)$. Não foi observada melhora da $\mathrm{AV}$ após o uso de lentes de contato em 8 casos $(18,61 \%)$. Seis pacientes $(13,95 \%)$ apresentaram um ganho de 5 linhas na tabela de Snellen. Três $(6,97 \%)$ pacientes que apresentavam AV de conta dedos a 50 cm pas-saram a ter AV 20/30, 20/40 e 20/60 (Gráfico 2). Dentre os pa-cientes que experimentaram melhora da AV a maioria o fez em número de 2 linhas ( 8 casos - 18,61\%).

Em relação às lentes de contato prescritas, observamos que seus valores dióptricos variaram nas lentes positivas de $+0,75$ (um caso de leucoma) a $+20,0$ (afacia). A variação nas lentes negativas foi menor, de $-0,50$ (perfuração corneana) a - 4,75 (afacia com ceratocone associados). O diâmetro médio das lentes de contato foi de 9,5 mm, variando de $8,5 \mathrm{~mm}$ (leucoma secundário a perfuração corneana) a $14,5 \mathrm{~mm}$ (afacia), com desvio-padrão de 0,87. Esta última lente era do

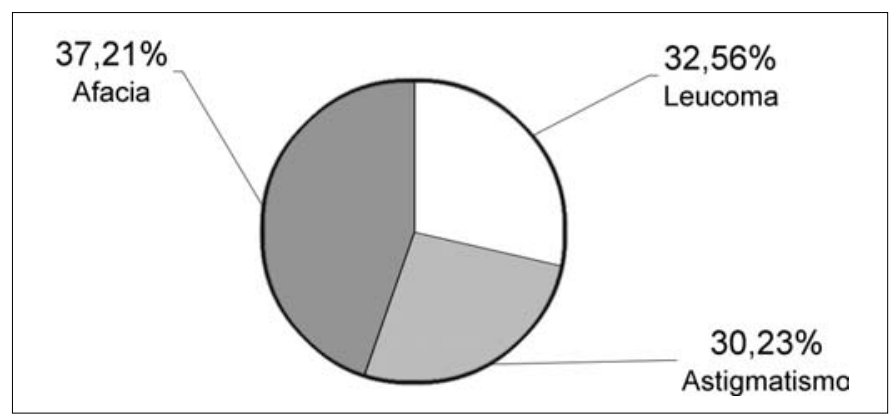

Gráfico 1 - Diagnósticos

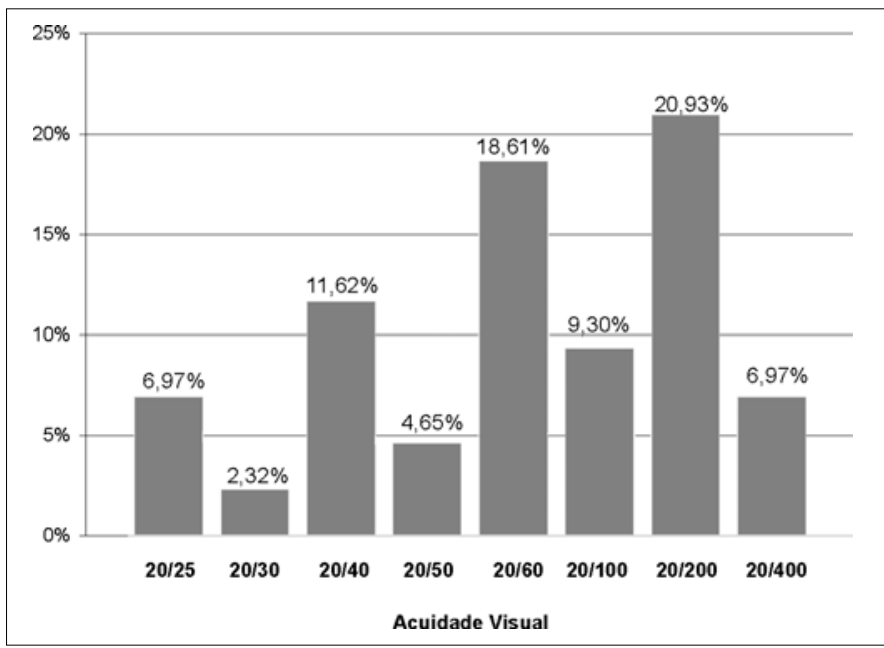

Gráfico 2 - Acuidade visual pós-trauma. Para melhor visualização da tabela excluimos as acuidades de conta dedos (CD) e movimento de mãos junto ao rosto (MM). São elas: CD $2 \mathrm{~m}(2,32 \%)$, CD a $1 \mathrm{~m}(2,32 \%)$, CD a $50 \mathrm{~cm}(9,30 \%)$, e MM $(2,32 \%)$ 


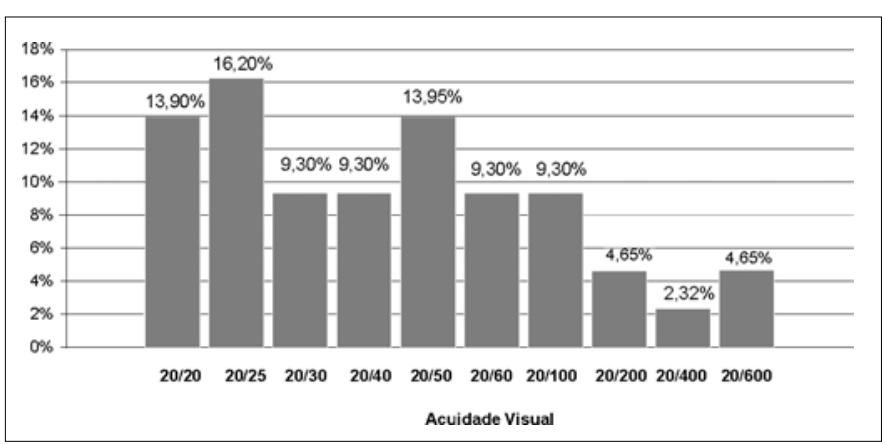

Gráfico 3 - Acuidade visual após lente de contato

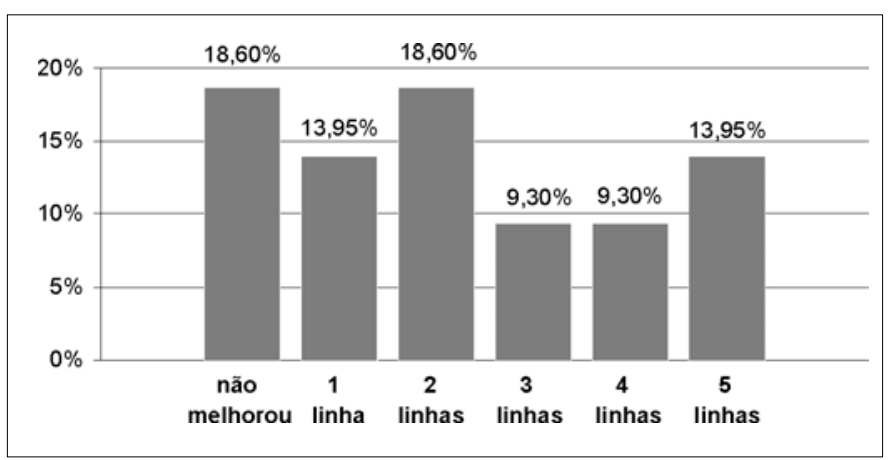

Gráfico 4 - Ganhos mais freqüentes de acuidade visual após uso de lentes de contato. Para melhor visualização gráfica foram excluídos os seguintes dados de melhora da AV: CD a $50 \mathrm{~cm}$ para $20 / 30, C D$ a $50 \mathrm{~cm}$ para $20 / 40(2,32 \%), C D$ a $50 \mathrm{~cm}$ para $20 / 60$, CD a $2 \mathrm{~m}$ para $20 / 100$ $(2,32 \%)$, MM para $20 / 100(2,32 \%)$, CD a $50 \mathrm{~cm}$ para $20 / 600(2,32 \%)$, CD a $1 \mathrm{~m}$ para $20 / 800(2,32 \%)$

tipo gelatinosa o que explica o seu diâmetro elevado. Os diâmetros mais utilizados foram: 9,5 mm em 14 casos $(32,56 \%)$, $9,8 \mathrm{~mm}$ em 8 casos $(18,61 \%)$ e $9,7 \mathrm{~mm}$ em 5 casos $(11,62 \%)$. O raio de curvatura das lentes prescritas variou de $6,40 \mathrm{~mm}$ (afacia e ceratocone associados) a $9,12 \mathrm{~mm}$ (perfuração corneana), com média de $8,0 \mathrm{~mm}$ e desvio-padrão de 0,60 . A curvatura média encontrada foi de $42,75 \mathrm{D}$, variando de $37,00 \mathrm{D}$ (perfuração corneana) a $52,75 \mathrm{D}$ (afacia e ceratocone associados), com desvio-padrão de 3,33. Quarenta e uma lentes $(95,35 \%)$ utilizadas tinham DK 71 , sendo que nas demais foi escolhida uma lente com DK 45.

O intervalo médio entre o trauma e o encaminhamento ao Serviço de Lentes de Contato foi de 3,5 anos, com um desviopadrão de 5 anos e um intervalo de 3 meses a 20 anos. A adaptação foi considerada como adequada em todos os casos. Houve a necessidade de troca de lente em apenas um paciente. Foi descrito um caso de úlcera bacteriana, sendo esta a única complicação encontrada na amostra.

\section{DISCUSSÃO}

A maioria dos pacientes atendidos eram jovens do sexo masculino (idade média $=27$ anos) estando em concordância com a epidemiologia do trauma ocular ${ }^{(1-2)}$. Em verdade, qual- quer estudo a respeito de trauma ocular mostra uma incidência maior em homens. Estudos mostram envolvimento de indivíduos do sexo masculino em até $81 \%$ dos $\operatorname{casos}^{(2)}$. Isto reflete a maior exposição do homem a atividades profissionais e práticas esportivas de risco ao trauma ocular e a convivência em ambientes notadamente mais violentos ${ }^{(2)}$.

A perfuração ocular como principal mecanismo do trauma também foi observado em outros trabalhos ${ }^{(1-2)}$. Em 1992, foi feita uma revisão semelhante a esta, onde a perfuração corneana contribuiu com $55,8 \%$ dos diagnósticos de trauma ${ }^{(1)}$.

Algumas alterações concomitantes, porém não atribuídas ao trauma, foram notadas no momento da adaptação das lentes de contato tais como ceratocone e atrofia do epitélio pigmentar macular, não constituindo impedimento à adaptação. No entanto um caso de entrópio cicatricial e outro de luxação posterior do cristalino necessitaram de intervenção cirúrgica para que se tornasse possível a adaptação das lentes de contato.

A perda de AV pós-trauma é a grande complicação em longo prazo. Esta redução depende da intensidade e do mecanismo do trauma. A afacia, os leucomas corneanos e o astigmatismo resultam em baixa da acuidade visual. Neste estudo a maioria dos pacientes apresentava uma AV pós-trauma corrigida de 20/200 (20,93\%). Em uma revisão de 2.279 casos de trauma encontrou 20/100 como a melhor AV corrigida mais freqüente ${ }^{(2)}$. Outro estudo identificou que a maior parte de sua amostra tinha AV corrigida igual ou melhor a 20/40 em 18 $(34,6 \%)$ pacientes $^{(1)}$. Neste mesmo estudo apenas em 8 $(18,61 \%)$ pacientes a AV era igual ou pior a $20 / 100$, dado semelhante ao do presente estudo. Isto pode ser justificado pela maior gravidade dos casos que chegam ao Serviço de Urgência do Hospital São Geraldo com diagnóstico de perfuração ocular com ou sem afacia. Após medir a AV de 92 pacientes vítimas de perfuração ocular com diferentes graus de lesão Banomani et al encontrou visão de $20 / 400$ ou pior em $61,2 \%$ dos $\operatorname{casos}^{(3)}$.

As lentes de contato indiscutivelmente melhoram a AV na maioria dos casos pós-trauma. Neste estudo isto foi demonstrado pela $\mathrm{AV}$ mais observada após a adaptação, 20/25 (8 casos - $18,61 \%$ ). Até $83 \%$ dos casos de trauma alcançam visão de 20/40 ou melhor após o uso das lentes ${ }^{(4)}$. Um estudo retrospectivo de 24 pacientes mostrou que naqueles adaptados com lentes rígidas gás-permeáveis a melhora na $\mathrm{AV}$ foi de 2 linhas ou mais ${ }^{(5)}$. Em nosso estudo este mesmo valor foi observado em 8 casos (18,61\%), constituindo a maioria. Entretanto, em 6 pacientes $(13,95 \%)$ o ganho chegou a 5 linhas, sendo estes os melhores resultados obtidos. Na literatura encontramos resultados semelhantes com melhora bastante expressiva da AV, chegando a $20 / 70$ em até $58,2 \%{ }^{(2)}$.

Houve casos em que o uso de lentes de contato não melhorou a AV. Neste estudo isto foi observado em 18,61\% dos casos, representando a principal causa de falha na adaptação. A não melhora da $\mathrm{AV}$ pode ocorrer em até $62,5 \%$ dos casos, considerando apenas os casos de insucesso ${ }^{(1)}$. Provavelmente nestes casos fatores não refracionais interferem na reabilitação visual. 
As lentes rígidas gás-permeáveis (RGP) foram prescritas em todos os casos. Esta prevalência não encontra correspondência na literatura ${ }^{(1-3,6)}$. É possível encontrarmos uma variabilidade grande quanto ao tipo de lente de contato utilizada: gelatinosa ou rígida. Alguns pacientes começam o tratamento com lente rígida, optando posteriormente por lente gelatinosa $^{(3)}$. Em estudo realizado em afacia pós-trauma ocular as lentes RGP foram prescritas em apenas $36,4 \%$ dos $\operatorname{casos}^{(1)}$. A adaptação com lentes RGP pode proporcionar melhor AV do que lentes gelatinosas, onde até $77 \%$ dos pacientes adaptados com lente RGP podem alcançar AV de 20/30 ou melhor, confrontando com $41 \%$ daqueles com lente gelatinosa. A população atendida em nossa instituição e composta principalmente de indivíduos de baixa renda para quem o custo e a facilidade de conservação das lentes são fatores que influenciam a escolha do tipo de lente a ser prescrita, o que nos faz prescrever lentes RGP com maior freqüência.

A adaptação não foi adequada em apenas um caso, onde houve piora da AV (de 20/15 para 20/20), porém questiona-se se a AV obtida com a melhor correção após o trauma estava correta, mas de uma forma ou de outra o paciente manteve uma visão corrigida normal. A troca por outra lente ocorreu apenas uma vez pois a paciente fez a higiene das lentes com álcool a $70 \%$, levando a opacificação das lentes e assim inutilizando-a.

\section{CONCLUSÃO}

Os dados obtidos neste estudo evidenciam que as lentes de contato são um complemento importante na reabilitação visual de pacientes vítimas de trauma ocular. Em certos casos, quando bem adaptada, dispensa uma segunda intervenção cirúrgica além de restabelecer uma visão funcional reintegrando o paciente às suas atividades diárias.

\section{ABSTRACT}

Purpose: To describe the findings related to contact lenses use after ocular trauma in the past 6 years at the São Geraldo Eye Hospital. We emphasize the improvement of visual acuity with contact lenses. Methods: Chart review from the Contact Lens Service in the past 6 years. The information about age, gender, diagnosis, associated diseases, involved eye, pre- and post-trauma visual acuity, radius, curvature and of the lenses is described. Results: The main diagnosis was ocular perforation, in 25 cases $(44.6 \%)$, the left eye was involved in 25 cases $(58.2 \%)$. The most frequent post-trauma best corrected visual acuity was 20/200 (21\%). The average best correct visual acuity after contact lens use was 20/20 $(14 \%)$. The major improvement in terms of lines of Snellen table occurred in 6 patients (14\%) (5 lines). The average lens diameter was $9.5 \mathrm{~mm}$, the average radius was $8.0 \mathrm{~mm}$. The lens curvature ranged from 37.00 dioptries to 52.75 dioptries. Most of the lenses had a 71 oxygen permeability index. The lens fitting was successful in all cases. The mean interval between the trauma and the contact lens fitting was 3.5 years. Conclusion: The contact lenses are important in the late ocular trauma rehabilitation, providing significant improvement of visual acuity in most of the cases.

Keywords: Contact lenses; Eye injuries; Visual acuity; Ocular adaptation

\section{REFERÊE CIAS}

1. Araújo Filho A, Kwitko S, Uras R, Lipener C. Adaptação de lente de contato em afacia traumática unilateral [resumo]. Arq Bras Oftalmol [periódico em CD-ROM]; 1991;54(4).

2. McMahon TT, Devulapally J, Rosheim KM, Putz JL, Moore M, White S. Contact lens use after corneal trauma. J Am Optom Assoc 1997;68:215-24.

3. Banonami MTBC, Alves MR, José NK, Júnior NAS. Ferimento perfurante de globo ocular em adultos. Arq Bras Oftalmol 1980;43:81-7.

4. Boghani S, Cohen EJ, Jones-Marioneaux S. Contact lenses after corneal lacerations. CLAO J 1991;17:155-8.

5. Lima CA, Chagas AI, Holzchh N, José NK. Adaptação de lente de contato pós-perfuração corneana [abstract]. Arq Bras Oftalmol 1996;59(4):351.

6. Schapira E, Lipner C, Moraes NSB. Recuperação visual em perfuração ocular. Arq Bras Oftalmol 1996;59:504-6.

\title{
ABO ELETRÔNICO
}

\author{
Novo site
}

Acesso: $\quad$ fittp://www.abonet.com.br 